\title{
Performance analysis of stopping turbo decoder iteration criteria
}

\begin{abstract}
The invention of turbo codes has attracted many researchers to explore various fields regarding turbo codes since it provides better error rate performance compared to the existing codes. Good error rate performance gives a penalty to the complexity of the codes. It includes the complexity of decoding algorithm and iterative decoding. This paper reviews the history of turbo codes and its structures. This paper also discusses the turbo decoding stopping criteria algorithm and analyses the performance of fixed and cross-entropy (CE) based stopping criteria. From the results, both criteria fail to terminate early in low SNR. However, CE-based stopping criteria outperform the fixed stopping criterion at high SNR and able to save more iteration and delay. This leads to an energy saving preservation while maintaining the performance of turbo codes.
\end{abstract}

Keyword: Turbo codes; Turbo iteration; Stopping criterion; Cross-entropy 